\title{
Intrinsic Microbicidal Activity and Pulmonary Hypertension in Isolated Newborn Piglet Lungs
}

\author{
SHEWAN M. AZIZ, THOMAS H. PAULY, AND MARK N. GILLESPIE \\ University of Kentucky A. B. Chandler Medical Center, College of Pharmacy, Division of Pharmacology and \\ Experimental Therapeutics [S.M.A., M.N.G.], and College of Medicine, Department of Pediatrics, Division of \\ Neonatology/T.H.P., M.N.G.J, Lexington, Kentucky 40536-0082
}

\begin{abstract}
The lung appears to be one of the dominant sites of bacterial clearance from the blood of infant piglets. Part of the lung bacterial clearance involves activation of an oxygen radical bactericidal mechanism that may be central to induction of acute pulmonary hypertension. The present study determined whether this bactericidal activity was intrinsic to resident lung cells. Isolated piglet lung preparations perfused with blood-free salt solution were used to delineate the amount of group B streptococci (GBS) extracted and killed upon transit through pulmonary vasculature. Approximately $45 \%$ of infused GBS was deposited in the lung during a single pulmonary transit, whereas nearly $40 \%$ of the organisms sequestered in the lung were killed within a 30-min period. Pretreatment with dimethylthiourea, a scavenger of hydroxyl radical that inhibits GBS-induced pulmonary hypertension, attenuated both bacterial uptake and killing to similar extents. Along with its deposition in the lung, GBS also induced concentrationdependent increases in total pulmonary resistance, which were related principally to increases in upstream arterial resistance. Lung weight also increased in a concentrationdependent manner. Both the increase in total pulmonary resistance and lung weight were temporally related to elevation in perfusion medium content of the stable thromboxane degradation product, thromboxane $B_{2}$. Pretreatment with indomethacin, a prostaglandin $H$ synthase inhibitor, or sodium(E)-3[4-(1-imidazolyl-methyl)phenyl]-2propenoic acid a thromboxane synthase inhibitor, reduced GBS-induced pulmonary hypertension and edema. Infusion of thromboxane mimetic, 9,11-dideoxy-9 $\alpha, 11 \alpha$-epoxymethano prostaglandin $F 2 \alpha$, mimicked the ability of GBS to increase total pulmonary and upstream arterial resistances but not development of pulmonary edema. These results suggest that, in isolated piglet lungs, GBS evokes an intrinsic bactericidal response residing within lung cells, probably pulmonary intravascular macrophages, which may be responsible for the initiation of pulmonary hemodynamic changes. It also appears that oxygen radicals play a major role in the bactericidal mechanism directed against GBS. In addition, these results suggest that thromboxane may be the major arachidonic acid metabolite responsible for GBS-induced pulmonary hypertension but perhaps not for development of pulmonary edema. (Pediatr Res 34: 3237, 1993)
\end{abstract}

Received July 13, 1992; accepted February 11, 1993.

Correspondence: Mark N. Gillespie, Ph.D., Division of Pharmacology and Experimental Therapeutics, College of Pharmacy, University of Kentucky A. B. Chandler Medical Center, Lexington, KY 40536-0082.

Supported in part by grants from the National Institutes of Health (HL-02055) and the American Heart Association, Kentucky affiliate.

\section{Abbreviations}

GBS, group B streptococcus

DMTU, 1,3-dimethyl-2-thiourea

KHS, modified Krebs-Henseleit solution

PIM, pulmonary intravascular macrophage

Ppa, mean pulmonary arterial pressure

Pla, left atrial pressure

Pdo, double occlusion pressure (equilibrium pressure)

$Q$, perfusion medium flow rate

$\mathrm{Ra}$, arterial resistance

$R v$, venous resistance

TX, thromboxane

TPR, total pulmonary resistance

cfu, colony-forming unit

OKY-046, sodium(E)-3[4-(1-imidazolyl-methyl)phenyl]-2propenoic acid

HBSS, Hanks' balanced salt solution

U44069, 9,11-dideoxy-9 $\alpha, 11 \alpha$-epoxymethano prostaglandin $\mathrm{F} 2 \alpha$

A serious complication of neonatal GBS infection is pulmonary hypertension $(1,2)$. Although studies in animal models of GBS sepsis have incriminated various chemical mediators in the pulmonary hypertensive response, including $\mathrm{TXA}_{2}$ (3), leukotrienes (4), toxic oxygen radicals (5), and tumor necrosis factor (6), much remains to be learned regarding the pathogenesis of lung injury in this setting. For example, the specific mechanism whereby bacteria trigger elaboration of vasoactive mediators has yet to be determined. We and others have proposed that bacteriaor particulate-induced activation of an oxygen radical-dependent microbicidal mechanism residing within PIM is a key stimulus for mediator release and acute pulmonary hypertension (7). In support of this contention, we have shown that a substantial proportion of GBS deposits in the lungs of intact infant piglets, where approximately half of the organisms are nonviable within a 60 -min period $(7,8)$. Interestingly, the severity of the pulmonary hypertensive response was inversely related to the percentage of bacteria remaining alive in the lung. The hydroxyl radical scavenger, DMTU, attenuates bacterial killing, TX accumulation, and pulmonary hypertension $(7,9)$. Electron microscopic analysis indicated that GBS were associated exclusively with PIM; evidence for interactions with other lung cells, including endothelial cells and leukocytes, during the acute phase of pulmonary hypertension was not found (8).

Evidence supporting the hypothesis that activation of oxygen radical-dependent bactericidal mechanisms is a key event in initiation of acute pulmonary hypertension has been derived largely from experiments in intact, anesthetized animals. Although we have previously demonstrated that GBS do indeed promote hypertension in isolated piglet lungs (8), it is not known whether lung cells mount a pathophysiologically relevant microbicidal response to intravascular bacteria or whether cell 
populations remote from the lungs and/or circulating blood cells kill bacteria which then localize in the lung and evoke mediator release and attendant hemodynamic abnormalities. Against this background, in the present study, we used an isolated piglet lung preparation to examine the intrinsic bactericidal activity directed at intravascular GBS and to determine whether the isolated lung responds to GBS in a manner similar to the intact pulmonary circulation.

\section{MATERIALS AND METHODS}

Drugs and chemicals. The following drugs and chemicals were used: DMTU (Aldrich Chemical Co., Milwaukee, WI) was dissolved in sterile isotonic saline on the day of each experiment. OKY-046 (Ono Pharmaceuticals, Inc., Osaka, Japan) was dissolved in double-distilled water on the day of the experiment. Indomethacin (Sigma Chemical Co., St. Louis, MO) was dissolved in $0.2 \mathrm{M} \mathrm{Na}_{2} \mathrm{CO}_{3}$. The TX mimetic, U44069 (Sigma), was dissolved in $95 \%$ ethanol at a concentration of $10 \mathrm{mg} / \mathrm{mL}$ and stored at $-70^{\circ} \mathrm{C}$. Aliquot parts were removed on the day of experimentation and diluted in saline. $\mathrm{TXB}_{2}$ antibody for RIA was kindly provided by Dr. H.-H. Tai (University of Kentucky, Lexington, $\mathrm{KY}$ ) and ${ }^{125} \mathrm{I}-\mathrm{TXB}_{2}$ was purchased from New England Nuclear (Boston, MA).

Preparation of GBS. GBS (Streptococcus agalacticae, serotype II) were obtained from American Type Culture Collection (no. 13813, Difco Laboratories, Detroit, MI). The bacteria were inoculated in brain-heart infusion broth containing $7 \%$ heat-inactivated FCS and grown to log phase at $37^{\circ} \mathrm{C}$ under a $5 \% \mathrm{CO}_{2}$ atmosphere. The medium was centrifuged at $2000 \times g$ for 15 min, and the pellet washed and resuspended in HBSS. The concentration of bacteria in the slurry was determined by quantitative culture and by relating OD to cfu.

Isolated piglet lung preparation. Thirty-eight piglets ranging in age and weight from 2 to $12 \mathrm{~d}$ and 2 to $4.5 \mathrm{~kg}$, respectively, were used in these studies. Each animal was anesthetized with an intraperitoneal injection of $30 \mathrm{mg} / \mathrm{kg}$ sodium pentobarbital. A tracheostomy was performed, and the animals were connected to a small-animal ventilator (model 670D; Harvard Apparatus Co., Millis, MA): Animals were ventilated at 50 breaths $/ \mathrm{min}$ with a mixture of $95 \%$ air and $5 \% \mathrm{CO}_{2}$ using a tidal volume of $7 \mathrm{~mL} / \mathrm{kg}$ and an end expiratory pressure of $4 \mathrm{~cm} \mathrm{H} \mathrm{H}_{2} \mathrm{O}$. A median sternotomy was performed, and $400 \mathrm{IU} / \mathrm{kg}$ heparin (Elkins-Sinn, Inc., Cherry Hill, NJ) were injected into the right ventricle. A 3$\mathrm{mm}$ glass cannula attached to flexible Tygon tubing (Fisher Scientific Co., Akron, $\mathrm{OH}$ ) was inserted into the left atrium via the left ventricle and ligated into place with umbilical tape. A similar cannula was inserted into the pulmonary artery via an incision in the right ventricular outflow tract. This cannula was secured with a tie through the transverse sinus so that the aorta was also ligated. The pulmonary artery cannula was immediately connected to a circuit, and pulmonary circulation was flushed at a constant flow $(30 \mathrm{~mL} / \mathrm{kg}$ body weight $/ \mathrm{min})$ in a nonrecirculating manner with $1 \mathrm{~L}$ of warmed $\left(37^{\circ} \mathrm{C}\right)$ and pH-adjusted (7.4) modified KHS (composition given below) using a peristaltic pump (model 60648; Cole Parmer Instrument Co., Chicago, IL). This washout procedure reduced neutrophil and platelet counts in the pulmonary effluent to less than $5 \%$ of normal values. Perfusion was then interrupted for $5 \mathrm{~min}$ while the heart and lungs were excised from the thorax, placed in a warmed $\left(37^{\circ} \mathrm{C}\right)$ humidified chamber, and suspended from a spring counterbalanced Grass FT03 tension transducer (Grass Instrument Co., Quincy, MA) that allowed a continuous measurement of lung preparation weights. The left atrial cannula was connected, and closed-circuit perfusion at the previous flow rate was then resumed with $500 \mathrm{~mL}$ of KHS. Along with lung weight, Ppa and $\mathrm{Pla}$ were measured continuously with Statham pressure transducers (Gould, Inc., Oxnard, CA) in conjunction with a Grass model 79 polygraph. Perfusate samples drawn from the left atrial cannula were analyzed for $\mathrm{pH}, \mathrm{PCO}_{2}$, and $\mathrm{PO}_{2}$ with an Instrument
Laboratories (Oxnard, CA) blood gas analyzer and maintained at 7.35 to $7.5,35$ to $45 \mathrm{~mm} \mathrm{Hg}$, and 120 to $150 \mathrm{~mm} \mathrm{Hg}$, respectively. Each preparation was perfused for 30 minutes before the experiment to verify stability.

Lung microvascular pressure was measured using the double occlusion technique. In brief, three-way solenoid valves were placed in the pulmonary arterial line, upstream from the arterial pressure transducer, and in the pulmonary venous line, downstream from the left atrial pressure transducer. By closing these valves simultaneously, equilibrium pressure, or Pdo, could be measured within the system. Because of the close agreement between Pdo and microvascular pressure determined by micropuncture (10) or by the isogravimetric technique (11), Pdo is taken as an estimate of microvascular pressure. Double occlusion was maintained for $4 \mathrm{~s}$, and then flow was allowed to resume. Use of the double occlusion method allowed partitioning of TPR into arterial or upstream resistance $(\mathrm{Ra})$ and venous or downstream resistance $(\mathrm{Rv})$, which were calculated in the following manner:

$$
\begin{aligned}
& \mathrm{TPR}=(\mathrm{Ppa}-\mathrm{Pla}) /(\mathrm{Q} / \mathrm{kg}) \\
& \mathrm{Ra}=(\mathrm{Ppa}-\mathrm{Pdo}) /(\mathrm{Q} / \mathrm{kg}) \\
& \mathrm{Rv}=(\mathrm{Pdo}-\mathrm{Pla}) /(\mathrm{Q} / \mathrm{kg})
\end{aligned}
$$

The perfusion medium, KHS, consisted of $\mathrm{NaCl}$ (Fisher Scientific Co., Pittsburgh, PA), $0.118 \mathrm{M} ; \mathrm{KCl}$ (Fisher Scientific), 4.7 $\times 10^{-3} \mathrm{M} ; \mathrm{CaCl}_{2}$ (Fisher Scientific), $1.9 \times 10^{-3} \mathrm{M} ; \mathrm{MgSO}_{4}$ (Fisher Scientific), $1.0 \times 10^{-3} \mathrm{M} ; \mathrm{KH}_{2} \mathrm{PO}_{4}$ (Fisher Scientific), $1.1 \times 10^{-3}$ $\mathrm{M} ; \mathrm{NaHCO}_{3}$ (Fisher Scientific), $2.5 \times 10^{-3} \mathrm{M}$; glucose (Fisher Scientific), $6.0 \times 10^{-3} \mathrm{M}$; and BSA (Sigma), 3\% (wt/vol).

The isolated, perfused piglet lung preparation was used in two general protocols, each lasting $<90 \mathrm{~min}$. Pilot studies indicated that the preparation was stable for at least this duration. The first series of experiments, as detailed below, determined whether the isolated lung mounted a bactericidal attack against GBS and whether killing of GBS could be ascribed in part to an oxygen radical-dependent mechanism. A second series of experiments characterized the effect of GBS on segmental pulmonary vascular resistance and edema formation and examined the potential involvement of oxygen radicals and TX, two mediators that have been implicated in GBS-induced pulmonary hypertension in intact animals $(3,5)$. In these latter studies, the inhibitory agents indomethacin, OKY -046, and DMTU, or their respective vehicles, were added to the perfusate reservoir to achieve final concentrations of $2.1 \times 10^{-5} \mathrm{M}, 2 \times 10^{-4} \mathrm{M}$, and $5 \times 10^{-4} \mathrm{M}$, respectively, and allowed to recirculate for $10 \mathrm{~min}$ before challenge with the indicated doses of GBS.

$R I A$ of $T X B_{2}$. Samples $(1 \mathrm{~mL})$ for RIA of $\mathrm{TXB}_{2}$ were drawn from the perfusate reservoir of isolated lung preparations during the baseline period and sequentially through the experiments. In studies assessing the effect of GBS on perfusate $\mathrm{TXB}_{2}$, samples were withdrawn before and approximately $5 \mathrm{~min}$ after bolus injections of the indicated concentrations of bacteria when perfusion pressures had returned to baseline or near-baseline levels. Samples were collected in $13 \times 100-\mathrm{mm}$ test tubes containing indomethacin (final concentration, $10^{-5} \mathrm{M}$ ) and stored at $-70^{\circ} \mathrm{C}$. $\mathrm{TXB}_{2}$ levels were measured by RIA as described previously (9).

Determination of GBS deposition and viability. To determine whether the isolated lung mounted a bactericidal attack against intravascular GBS, we quantified extraction of the organism during transit through pulmonary vasculature and assessed viability of the organism in lung tissue $30 \mathrm{~min}$ after termination of bacterial infusion. Our strategy was to determine the total bacteria delivered to the lung $\left(\mathrm{cfu}_{\mathrm{in}}\right)$ and the total bacteria recovered in lung effluent $\left(\mathrm{cfu}_{\mathrm{out}}\right)$; the difference between these two values (cfu deposited) reflects the abundance of organisms sequestered in the lung, providing no bacterial growth or killing occurred in the perfusion medium. If, for example, bacteria proliferated in the perfusion medium, then the cfu recovered in the pulmonary 
effluent during the 30-min washout period after termination of the infusion would be spuriously elevated, which, in turn, would decrease calculated cfu deposited and, as described below, decrease the calculated bacterial killing. Accordingly, in preliminary experiments, we used quantitative bacterial culture to determine whether GBS proliferated over a 45 -min period when incubated in warmed, oxygenated KHS that either had been conditioned by pulmonary perfusion or was not exposed to the pulmonary circulation. GBS neither proliferated nor was killed when incubated in conditioned or nonconditioned KHS (data not shown), thus validating the mass-balance approach to quantifying lung bacterial disposition in the isolated lung preparation.

The following protocol was used to assess lung bacterial deposition. After a 30-min stabilization period, perfusion was switched to the nonrecirculating mode. Either saline (vehicle) or DMTU $\left(5.0 \times 10^{-4} \mathrm{M}\right)$ was added to the perfusate reservoir and allowed to recirculate for $10 \mathrm{~min}$. The preparation was then switched to the nonrecirculating mode, and GBS were infused at a rate of $10^{6} \mathrm{cfu} / \mathrm{mL} / \mathrm{min}$ for a $5-\mathrm{min}$ period, during which time the pulmonary effluent was collected. Subsequently, the pulmonary circulation was perfused in a nonrecirculating manner with bacteria-free KHS for another $5 \mathrm{~min}$, and this was also collected. Fresh, bacteria-free perfusate was then added and allowed to recirculate for an additional $25 \mathrm{~min}$ before its collection. The total amounts of GBS in the infusate and in the recirculated pulmonary effluents were determined by quantitative bacterial cultures. The percentage of deposition was determined as follows:

$$
\% \text { GBS deposition }=\frac{c f u_{\text {in }}-c f u_{\text {out }}}{c f u_{\text {in }}} \times 100
$$

For determination of bacterial viability, two 6-g samples were taken aseptically from the lung after the $25-\mathrm{min}$ recirculating perfusion period and placed in preweighed scintillation vials containing $4 \mathrm{~mL}$ of HBSS. These samples were homogenized at high speed for 1 min (Tissuemizer, Tekmar Co., Cincinnati, $\mathrm{OH})$, and aliquots were then streaked on $5 \%$ sheep blood agar plates and incubated for 24 to $48 \mathrm{~h}$ in $5 \% \mathrm{CO}_{2}$ at $37^{\circ} \mathrm{C}$. Plates were subsequently counted to determine the number of $\mathrm{cfu}$ and expressed as cfu/g of lung. The total viable GBS was determined by multiplying the cfu/g by total lung weight, while the percentage of GBS killing was determined as follows:

$$
\% \text { GBS killing }=\frac{\text { cfu deposited }-\mathrm{cfu} \text { viable }}{\mathrm{cfu} \text { deposited }} \times 100
$$

Statistical analysis. The impact of DMTU administration on uptake and killing of GBS was evaluated using an unpaired $t$ test. Effects of indomethacin and OKY-046 on TPR, TX generation, and lung weight in GBS-treated isolated piglet lungs were assessed using a one-way analysis of variance and Neuman-Kuels test for multiple comparisons. A one-way analysis of variance combined with Neuman-Kuels test also was used to examine the effect of increasing concentration of GBS on TPR, TXB $\mathrm{TXcu}_{2}$ accumulation, and lung weight.

\section{RESULTS}

GBS uptake and viability in isolated piglet lungs. The percentages of pulmonary uptake and killing of GBS after intravascular administration are shown in Figure 1. In control lungs, $44 \pm$ $4.7 \%$ of the GBS dose was cleared during a single pass through the pulmonary circulation. At $30 \mathrm{~min}$ after termination of infusion, $42 \pm 7 \%$ of the total GBS dose was killed. Additional lung preparations were treated with DMTU to determine whether hydroxyl or hypochlorous radicals contributed to the clearance mechanism in isolated lungs, as they appear to do in the intact animal. In the presence of DMTU, lung uptake and killing were $13 \pm 2.2 \%$, and $19.4 \pm 2.7 \%$, respectively, and were significantly reduced in comparison with control preparations.

Effect of $G B S$ on hemodynamics in isolated piglet lung prepa-

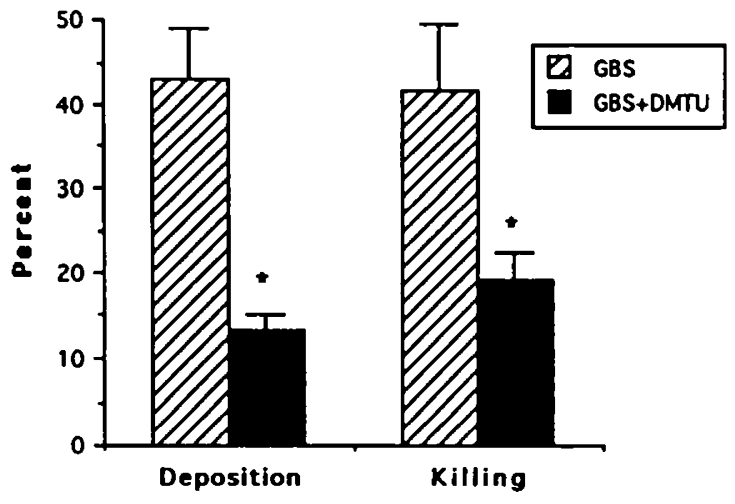

Fig. 1. Percentage of deposition and killing of GBS $\left(10^{6} \mathrm{cfu} / \mathrm{mL} / \mathrm{min}\right.$ for $5 \mathrm{~min}$ ) in isolated piglet lungs perfused with KHS in absence and presence of $10^{-3} \mathrm{M}$ DMTU. Each bar indicates the mean \pm SEM of six observations. *, Significantly different from GBS at $p<0.05$. See Materials and Methods for information regarding determination of bacterial deposition and viability.

rations. As shown in Figure 2, GBS provoked dose-dependent increases in TPR. Increases in TPR were related principally to elevations in Ra with only a modest contribution of increases in Rv. Along with the increase in TPR, GBS also caused dosedependent increases in weights of isolated piglet lungs (Fig. 2). The vehicle for GBS, HBSS, was without effect on vascular resistance in the isolated lung preparation (data not shown).

$T X$ in GBS-induced pulmonary hypertension. As shown in Figure 2, GBS caused concentration-dependent increases in the perfusion medium content of the stable TXA $\mathrm{T}_{2}$ metabolite, TXB 2 . HBSS, the vehicle for GBS, did not cause appreciable changes in the perfusate content of $\mathrm{TXB}_{2}$ (data not shown). Two approaches were used to assess the role of TX in GBS-induced pulmonary hypertension in infant piglet lungs. In the first, the impact of several interventions that disrupt TX synthesis in intact piglets, indomethacin, OKY -046 , and DMTU, on changes in TPR, lung weight, and perfusate $\mathrm{TXB}_{2}$ evoked by $10^{8} \mathrm{cfu} \mathrm{GBS} / \mathrm{mL}$ was assessed. As shown in Table 1, pretreatment with indomethacin abolished the GBS-induced elevation in TPR, the lung weight gain, and the increase in perfusate $\mathrm{TXB}_{2}$ content, whereas $\mathrm{OKY}$ 046 treatment suppressed GBS-induced increases in these parameters. Surprisingly, although DMTU prevented the rise in TPR and lung weight, the scavenger failed to suppress the increase in perfusate $\mathrm{TXB}_{2}$. A second approach used to examine the role of TX in GBS-induced pulmonary hypertension and lung weight gain was to determine whether the TX mimetic, U44069, mimicked the effect of GBS on segmental pulmonary vascular resistance and changes in lung weight. As illustrated in Figure 3, U44069 caused dose-related increases in TPR that were attributed to increases in $\mathrm{Ra}$ with only a modest contribution of increased Rv. Along with the pressor response, U44069 also produced concentration-dependent increases in lung weight (Fig. 3 ). To provide additional insight into the role of TX in GBSinduced increases in lung weight, the relations between GBSand U44069-induced increases in Pdo and lung weight are shown in Figure 4. At equivalent Pdo, GBS evoked greater increases in lung weight than U44069.

\section{DISCUSSION}

GBS deposits in the lungs of intact, newborn piglets, where the bacteria are apparently killed in part by an oxygen radicaldependent mechanism $(7,8)$. In this complicated preparation, it is not possible to determine whether lung cells mount a pathophysiologically relevant microbicidal response to intravascular bacteria or whether cell populations remote from the lungs and/ or circulating blood cells kill bacteria that then localize in the lung. The present study, therefore, used an isolated piglet lung 

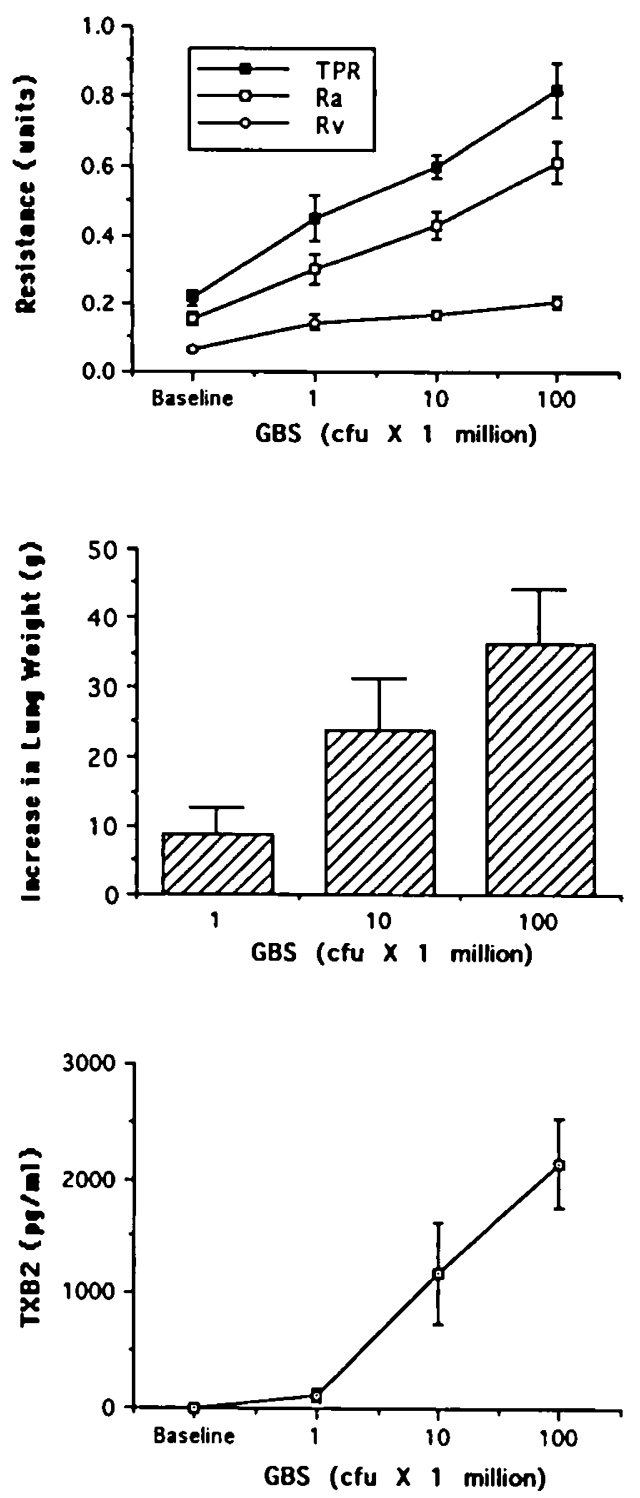

Fig. 2. Dose-dependent actions of GBS on segmental pulmonary vascular resistances (top), lung weight (middle), and perfusion medium content of $\mathrm{TXB}_{2}$ (bottom) in isolated piglet lungs perfused with KHS. Each point or bar reflects the mean \pm SEM of six observations. A oneway analysis of variance and Neuman-Kuels test indicated that GBS caused dose-dependent increases in all measured parameters.

preparation perfused with blood-free medium to examine the intrinsic bactericidal activity directed at intravascular GBS and to determine whether the isolated lung responds to GBS in a manner similar to the intact pulmonary circulation.

Results of the present experiments demonstrate that in saltsolution-perfused, isolated piglet lung preparations, almost $45 \%$ of the infused dose of GBS was cleared by the pulmonary circulation, and nearly $40 \%$ of the total cleared dose was killed by the lung. Because the pulmonary vasculature was thoroughly washed to eliminate circulating neutrophils and platelets, it is reasonable to suggest that isolated piglet lungs mount an intrinsic bactericidal attack that clears and kills the organism independent of blood-borne elements. The lung cells responsible for this bactericidal activity are unknown. Our previous electron microscopic analysis (8) suggested that GBS was localized in PIM with no evidence for bacterial interactions with either neutrophils or endothelial cells. This observation is consistent with reports from other laboratories. For example, Warner et al. (12-14) in a series of elegant studies in sheep, found that both Pseudomonas aeruginosa and iron oxide particles localized exclusively within PIM. Miyamoto et al. (15) reported a similarly restricted localization of inert microspheres in sheep lungs. PIM in vitro are phagocytic, although less so than alveolar macrophages (16), and generate substantial amounts of eicosanoids, including those incriminated in GBS-induced pulmonary hypertension, as well as various cytokines $(16,17)$. Collectively, these findings support the view that PIM play a major role in clearance of GBS as it transits the pulmonary vascular bed and that clearance of the bacteria is important for release of mediators that incite the hypertensive response and attract additional inflammatory cells (e.g. neutrophils) to the lung.

Treatment of isolated lungs with DMTU caused a significant reduction in both bacterial deposition and killing. In the present study, bacterial "killing" was normalized to the extent of bacterial deposition; the reduction in bacterial killing by DMTU can thus not be ascribed to the reduction in pulmonary bacterial sequestration unless a positive relationship exists between the process of killing and the density of GBS in the lung. We are not aware of evidence supporting such a relationship. At this point in our studies, we believe that the isolated lung, like the blood-perfused lung in the intact animal, mounts a bactericidal attack on intravascular GBS that is in part dependent on toxic oxygen radicals. Moreover, because of the relative selectivity of DMTU for hydroxyl radical $(18,19)$, it is reasonable to suggest that this free radical species figures prominently in pulmonary bactericidal activity directed against GBS.

As noted above, DMTU also reduced the extent of GBS deposited in the isolated lung. This finding differs from intact piglets, in which DMTU suppressed bacterial killing without effect on the extent of bacterial deposition (7). The exact mechanism by which DMTU reduced bacterial uptake in isolated lungs is unknown. Changes in delivery of the bacteria to the lung cannot be involved in this constant flow-perfused preparation. Inhibition of pulmonary vasoconstriction by DMTU (see below) also does not seem to be important, inasmuch as, in intact piglets, DMTU (9), OKY-046, and indomethacin (20) all prevent GBS-induced pulmonary hypertension without greatly affecting the extent of lung GBS deposition. Additional studies will be required to delineate the mechanism whereby DMTU retards GBS uptake in isolated, perfused piglet lungs.

Similar to its pulmonary hemodynamic effects in intact piglets, GBS evoked concentration-dependent increases in TPR, Pdo,

Table 1. Effect of prostaglandin H synthase or TX synthetase inhibition on GBS-induced pulmonary hypertension and edema in isolated piglet lungs

\begin{tabular}{lccc}
\hline \multicolumn{1}{c}{ Treatment } & $\begin{array}{c}\text { Increase in } \mathrm{TXB}_{2} \\
(\mathrm{pg} / \mathrm{mL})\end{array}$ & $\begin{array}{c}\text { Increase in Rt } \\
\text { (units) }\end{array}$ & Increase in lung wt (g) \\
\hline GBS alone & $2150 \pm 380$ & $0.6 \pm 0.06$ & $36.03 \pm 8.20$ \\
GBS + indomethacin* & $-1 \pm 19.7 \dagger$ & $0 \dagger$ & $0.15 \pm 0.15 \dagger$ \\
GBS + OKY-046* & $218 \pm 47.9 \dagger$ & $0.11 \pm 0.06 \dagger$ & $0.55 \pm 0.38 \dagger$ \\
GBS + DMTU* & $1674.6 \pm 392$ & $0.17 \pm 0.07 \dagger$ & $5.96 \pm 1.90 \dagger$ \\
\hline
\end{tabular}

\footnotetext{
* Indomethacin. OKY-046, or DMTU was added to the perfusate reservoir to achieve final concentrations of $2.1 \times 10^{-5} \mathrm{M}, 2.0 \times 10^{-4} \mathrm{M}$, and $5.0 \times 10^{-4} \mathrm{M}$. respectively, and allowed to recirculate for $10 \mathrm{~min}$ before infusion of GBS at a rate of $10^{6} \mathrm{cfu} / \mathrm{mL} / \mathrm{min}$. Each of the pharmacologic probes was studied in four to six isolated lung preparations. $\mathrm{Rt}$, total pulmonary vascular resistance.

+ Different from GBS alone at $p<0.01$.
} 



Fig. 3. Dose-dependent actions of the TX mimietic, U44069, on segmental pulmonary vascular resistances (top) and lung weight (bottom) in isolated piglet lungs perfused with KHS. Concentrations of U44069 in $\mathrm{mol} / \mathrm{L}$ are $0.0456,0.1,0.185,0.35,0.7,1.4,2.8$, and $5.7 \times 10^{-9} \mathrm{M}$. Each point or bar reflects the mean \pm SEM of four observations. A oneway analysis of variance and Neuman-Kuels test indicated that U44069 caused dose-dependent increases in all measured parameters.



Fig. 4. Relation between double occlusion pressure $(P d o)$ and increases in lung weight for isolated piglet lung preparations challenged with GBS or U44069. These curves were constructed with values for Pdo used for calculation of segmental pulmonary vascular resistances in the top panels of Figures 2 and 3 and lung weight data from the middle and bottom panels of Figures 2 and 3, respectively.

and lung weight in isolated, KHS-perfused lungs. The increase in TPR resulted primarily from increases in $\mathrm{Ra}$, with modest increases in Pdo and Rv. Multiple lines of evidence suggest that the initial pulmonary hypertension in GBS sepsis is due to arachidonic acid metabolites, particularly $\mathrm{TXA}_{2}$. Temporal associations between increases in $\mathrm{TXB}_{2}$ and increases in pulmonary vascular resistance have been reported in animals infected with GBS (21). Pretreatment with either cyclooxygenase inhibitors or specific TX synthase inhibitors prevents or reverses GBS-induced pulmonary hypertension and the increase in TX generation (3, 22). Based on this evidence, a series of experiments was designed to test whether TX mediated the response to GBS in isolated piglet lungs. In support of this contention, we found that the dose-dependent increase in vascular resistance and lung weight evoked by GBS in isolated piglet lungs was accompanied by accumulation of $\mathrm{TXB}_{2}$ in the perfusion medium. Pretreatment with indomethacin, a prostaglandin $\mathrm{H}$ synthase inhibitor, or OKY-046, an inhibitor of TX synthesis, suppressed GBS-induced increases in TPR, the accumulation of $\mathrm{TXB}_{2}$ in the perfusion medium, and the lung weight gain. Finally, U44069, an epoxymethano analog of the cyclic endoperoxides believed to act on the TX receptor, mimicked the effect of GBS on TPR and, more importantly, on segmental vascular resistance. In this latter context, U44069, like GBS, was found to increase PVR through elevation of Ra. The newborn piglet lung thus behaves more like isolated rat lungs, in which $\mathrm{TXA}_{2}$ increases resistance through an effect on upstream vessels (23), than the dog lung, in which the eicosanoid acts as a venoconstrictor (24).

Clearly, however, TX is not the sole chemical mediator of the response to GBS. Other investigators have shown that lipoxygenase products of arachidonic acid metabolism contribute to hemodynamic effects of GBS on the newborn lung (4). More recent studies have incriminated cytokines such as tumor necrosis factor (6). In the present experiments, we found that U44069 did not mimic the effect of GBS on pulmonary edema formation; at equivalent Pdo, the lung weight gain caused by U44069 was less than that provoked by GBS. This observation may be significant for two reasons. First, along with the evidence noted above, it provides additional support for the contention that mediators in addition to TX contribute to the response to GBS in this preparation. Second, and in a related context, GBS may promote lung water accumulation by a mechanism involving increased pulmonary endothelial permeability and not solely increased capillary hydrostatic pressure.

DMTU, like indomethacin and OKY-046, prevented GBSinduced pulmonary hypertension, but in the case of the hydroxyl radical scavenger, inhibition of the pulmonary pressor response was not associated with diminution of TX accumulation. This surprising observation differs from our previous finding in intact piglets that DMTU abolished the rise in $\mathrm{TXB}_{2}$ along with the pulmonary hypertension (9) and casts doubt on the hypothesis that the induction of TX synthesis in GBS-treated piglets is oxygen radical-dependent. At present, we cannot explain the disparity between the effect of DMTU in intact piglets and isolated piglet lungs. Perhaps in the intact animal, endogenous scavenger systems act in concert with DMTU to reduce the oxygen radical burden in a cellular compartment involved with arachidonic acid metabolism; in the absence of these other scavenger mechanisms, DMTU may not be sufficient to reduce the oxygen radical burden below a critical level. The question also may be raised as to the mechanism by which DMTU prevented GBS-induced pulmonary hypertension in the absence of any decrement in perfusate TX levels. We suspect that inhibition of GBS-induced pulmonary hypertension in the isolated preparation may be related to protection against the breakdown of endothelium-derived relaxing factor. Oxygen radicals are known to degrade endothelium-derived relaxing factor (25). Consequently, DMTU, by decreasing the burden of oxygen radicals, could be expected to magnify the impact of endothelium-derived relaxing factor on the pulmonary circulation and thereby prevent GBS-induced vasoconstriction. Additional studies will be required to explain the differences between effects of DMTU on the isolated lung and intact animal and to determine the mechanism whereby the free radical scavenger protects the isolated lung from GBS-induced pulmonary hypertension.

In summary, results of the present study suggest that the 
isolated piglet lung mounts an oxygen radical-dependent bactericidal response to GBS. Interactions between GBS and resident lung cells, probably PIM, are associated with development of pulmonary hypertension. In addition, TX seems to be the major arachidonic acid metabolite responsible for GBS-induced pulmonary hypertension, but other chemical mediators probably contribute to pulmonary edema formation. The isolated piglet lung preparation may prove useful for examining intrinsic pulmonary bacterial clearance mechanisms in health and disease and other related issues.

\section{REFERENCES}

1. Shankaran S, Farooki ZQ, Desai R $1982 \beta$-Hemolytic streptococcal infection appearing as persistent fetal circulation. Am J Dis Child 136:725-727

2. Walsh JA, Hutchins S 1990 Group B streptococcal disease: its importance in the developing world and prospect for prevention with vaccines. Pediatr Infect Dis 8:271-276

3. Gibson RL, Truog WE, Redding GI 1987 Thromboxane-associated pulmonary hypertension during three types of gram-positive bacteremia in piglet. Pediatr Res 23:553-556

4. Goldberg RN, Suguihara C, Streitfeld MM, Bancalari A, Clark MR, Bancalari E 1986 Effect of a leukotriene antagonist on the early hemodynamic manifestations of group B streptococcal sepsis in piglets. Pediatr Res 20:1004 1008

5. Pauly TH, Bowdy BD, Haven CA, Barr SB, Gillespie MN 1988 Evidence for hydroxyl radical involvement in group B streptococcus-induced pulmonary hypertension and arterial hypoxemia in young piglets. Pediatr Res 24:739745

6. Gibson RL, Redding GJ, Henderson WR, Truog WE 1991 Group B streptococcus induces tumor necrosis factor in neonatal piglets: effect of the tumor necrosis factor inhibitor pentoxyfylline on hemodynamics and gas exchange. Am Rev Respir Dis 143:598-604

7. Bowdy BD, Marple SL, Pauly TH, Coonrod JD, Gillespie MN 1990 Oxygen radical dependent bacterial killing and pulmonary hypertension in piglets infected with group B streptococcus. Am Rev Respir Dis 141:648-653

8. Bowdy BD, Marple SJ, Aziz SM, Yoneda K, Coonrod JD, Gillespie MN 1990 Organ specific deposition of group B streptococci in infant piglets: evidence that pulmonary hypertension results from a direct interaction with target cell in the lung. Pediatr Res 27:344-348

9. Shook LA, Pauly TH, Marple SL, Horstman SJ, Tai H-H, Bowdy BD, Gillespie MN 1990 Group B streptococcus promotes oxygen radical-dependent thromboxane accumulation in young piglets. Pediatr Res 27:349-352

10. Nagasaka Y, Bhattacharya J, Nanjo S, Gropper MA, Staub CN 1984 Micro- puncture measurement of lung microvascular pressure profile during hypoxia in cats. Circ Res 54:90-96

11. Townsley MI, Korthins JR, Parker CJ, Taylor EA 1986 Validation of double occlusion method for Pc,i in lung and skeletal muscle. J Appl Physiol 61:127132

12. Warner AE, Monila RM, Brain JD 1987 Uptake of bloodborne bacteria by pulmonary intravascular macrophages and consequent inflammatory response in sheep. Am Rev Respir Dis 136:683-690

13. Warner AE, Brain JD 1986 Intravascular pulmonary macrophage, a novel cell removes particles from blood. Am J Physiol 250:R728-R732

14. Warner AE, Barry BE, Brain JD 1986 Pulmonary intravascular macrophages in sheep. Morphology and function of a novel constituent of the mononuclear phagocyte system. Lab Invest 55:276-288

15. Miyamoto K, Schultz E, Heath T, Mitchell MD, Albertine KH, Staub NC 1988 Pulmonary intravascular macrophages and hemodynamic effects of liposomes in sheep. J Appl Physiol 64:1143-1152

16. Chitko-McKown CG, Chapes SK, Brown RE, Phillips RD, McKown RD, Blecha F 1991 Porcine alveolar and pulmonary intravascular macrophages: comparison of immune functions. J Leuk Biol 50:364-372

17. Bertram TA, Overby AH, Danilowicz R, Eling TE, Brody AR 1988 Pulmonary intravascular macrophages metabolize arachidonic acid in vitro. Comparison with alveolar macrophages. Am Rev Respir Dis 138:936-944

18. Fox RB 1984 Prevention of granulocyte-mediated oxidant lung injury in rats by a hydroxyl radical scavenger, dimethylthiourea. J Clin Invest 74:14561464

19. Wasil M, Halliwell B, Grootveld M, Moorehouse CP, Hutchison DCS, Baum H 1988 The specificity of thiourea, dimethylthiourea, and dimethylsulfoxide as scavengers of hydroxyl radicals. Their protection of $\alpha 1$-antiproteinase against inactivation by hypochlorous acid. Biochem $J$ 243:867-870

20. Pauly TH, Aziz SM, Horstman SA, Gillespie MN 1991 Impact of cyclooxygenase and thromboxane synthase blockade on lung microbicidal activity directed against group B streptococci in infant piglets. Pediatr Res 31:14-17

21. Runkle B, Goldberg RN, Streifeld MM, Clark MR, Buron E, Setzer ES, Bancalari E 1984 Cardiovascular changes in group B streptococcal sepsis: relationship to prostacyclin and thromboxane $\mathrm{A}_{2}$. Pediatr Res 18:874-878

22. Truog WE, Sorenson GK, Standaert TA, Redding GJ 1986 Effects of a thromboxane synthesis inhibitor, damzegrel (UK 38,485 ) on pulmonary gas exchange and hemodynamics in neonatal sepsis. Pediatr Res 20:486-491

23. Barnard JW, Ward RA, Adkins WK, Taylor AE 1992 Characterization of thromboxane and prostacyclin effects on pulmonary vascular resistance. $J$ Appl Physiol 72:1845-1853

24. Barman SA, Senteno E, Smith S, Taylor AE 1989 Acetylcholine effect on vascular resistance and compliance in the pulmonary circulation. J Appl Physiol 67:1495-1503

25. Gryglewski JR, Palmer JMR, Moncada S 1986 Superoxide anion is involved in the breakdown of endothelium derived vascular relaxing factor. Nature $320: 454-456$ 